Pacific Journal of Mathematics

ON FREDHOLM TRANSFORMATIONS IN YEH-WIENER 


\section{ON FREDHOLM TRANSFORMATIONS IN YEH-WIENER SPACE}

\section{CHULL PARK}

Let $C_{Y}$ denote the Yeh-Wiener space, i.e., the space of all real-valued continuous functions $f(x, y)$ on $I^{2} \equiv[0,1] \times[0,1]$ such that $f(0, y)=f(x, 0) \equiv 0$. Yeh has defined a Gaussian probability measure on $C_{Y}$ such that the mean of the process

$$
m(x, y) \equiv \int_{C_{Y}} f(x, y) d_{Y} f=0
$$

and the convariance

$$
R(s, t, x, y) \equiv \int_{C_{Y}} f(s, t) f(x, y) d_{Y} f=(1 / 2) \min (s, x) \min (t, y) .
$$
the form

Consider now a linear transformation of $C_{Y}$ onto $C_{Y}$ of

$$
\begin{aligned}
& T: f(x, y) \rightarrow g(x, y) \\
& \quad=f(x, y)+\int_{I^{2}} K(x, y, s, t) f(s, t) d s d t
\end{aligned}
$$

which is often called a Fredholm transformation. The main purpose of this paper is to find the corresponding RadonNikodym derivative thus showing how the Yeh-Wiener integrals transform under the transformation.

The transformations considered here contain the Volterra transformation

$$
T_{1}[f(x, y)]=f(x, y)+\int_{0}^{y} \int_{0}^{x} K(x, y, s, t) f(s, t) d s d t
$$

as a special case.

Such transformations in Wiener space have been studied a great deal by Cameron and Martin [1], Woodward [9], Segal [5], [6], and Shepp [7], and the results have proved very useful in the evaluation of various Wiener integrals.

The transformation theorems in this paper are based on stochastic integrals called the generalized Paley-Wiener-Zygmud (P.W.Z.) integrals given in [3] and [4]. For a function $h(x, y) \in L^{2}\left(I^{2}\right)$ and $f(x, y) \in C_{Y}$, the generalized P.W.Z. integral is defined to be

$$
\int_{I^{2}} h f d^{*} f \equiv \lim _{n \rightarrow \infty} \int_{I^{2}}(h f)_{n} d f
$$


where $(h f)_{n}$ is the $n^{\text {th }}$ partial sum of the Fourier expansion of $h(x, y) f(x, y)$ with respect to a C.O.N. set belonging to a class of C.O.N. systems $\left\{\alpha_{k}(x, y)\right\}$ with each $\alpha_{k}(x, y)$ of B.V. satisfying the condition

$$
\text { l.i.m. } \sum_{j=1}^{n} \alpha_{j}(x, y) \int_{0}^{y} \int_{0}^{x} \alpha_{j}(s, t) d s d t=\frac{1}{4},
$$

and the limit and on the right of (1.2) is an ordinary Riemann-Stieltjes (R-S) integral. It it known that the limit in (1.2) exists for almost all $f$ in $C_{Y}$ and it is essentially independent of the particular choice of the C.O.N. set in the class. (For details see [3] and [4].)

The Fredholm determinant $D(K)$ of $K(x, y, s, t) \in L^{2}\left(I^{4}\right)$ for $\lambda=$ -1 is defined by

$D(K) \equiv 1$

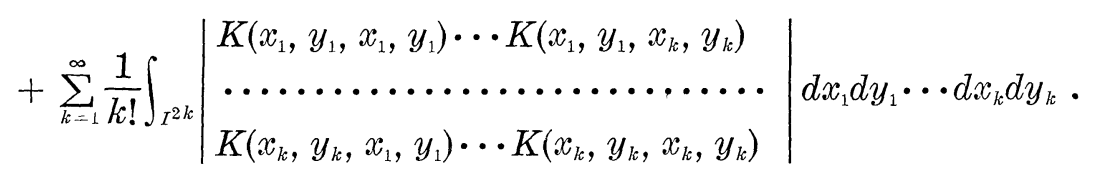

\section{Statement of main results.}

Theorem I. Suppose that each $K_{i}(x, y, s, t), i=1,2,3,4$, is continuous on $I^{4}$ and absolutely continuous in $x, y$ for each $(s, t) \in I^{2}$ and $K_{1}(0, y, s, t)=K_{1}(x, 0, s, t)=K_{3}(x, 0, s, t)=K_{4}(0, y, s, t) \equiv 0$. Let $K(x, y, s, t)$ be defined on $I^{4}$ by

(2.1) $K(x, y, s, t)= \begin{cases}K_{1}(x, y, s, t) \quad \text { if } x<s, y<t \\ K_{2}(x, y, s, t) \quad \text { if } x>s, y>t \\ K_{3}(x, y, s, t) \quad \text { if } x>s, y<t \\ K_{4}(x, y, s, t) \quad \text { if } x<s, y>t \\ 2^{-1}\left(K_{1}+K_{3}\right)(x, y, x, t) \quad \text { if } x=s, y<t \\ 2^{-1}\left(K_{2}+K_{4}\right)(x, y, x, t) \quad \text { if } x=s, y>t \\ 2^{-1}\left(K_{1}+K_{4}\right)(x, y, s, y) \quad \text { if } x<s, y=t \\ 2^{-1}\left(K_{2}+K_{3}\right)(x, y, s, y) \quad \text { if } x>s, y=t \\ 4^{-1}\left(K_{1}+K_{2}+K_{3}+K_{4}\right)(x, y, x, y) \quad \text { if } x=s, y=t,\end{cases}$

where $\left(K_{1}+K_{3}\right)(x, y, x, t)=K_{1}(x, y, x, t)+K_{3}(x, y, x, t)$, etc., and let

$$
\begin{aligned}
& \dot{\phi}(x, s, t) \equiv K\left(x, t^{+}, s, t\right)-K\left(x, t^{-}, s, t\right) \\
& \dot{\psi}(y, s, t) \equiv K\left(s^{+}, y, s, t\right)-K\left(s^{-}, y, s, t\right) .
\end{aligned}
$$

To be definite at each jump discontinuity, let us agree that the partials take left-hand limit whenever it fails to exist at a point, i.e., 


$$
\frac{\partial^{2}}{\partial y \partial x} K(x, y, s, t) \equiv H(x, y, s, t)=\lim _{(u, v) \rightarrow\left(x^{-}, y^{-}\right)} \frac{\partial^{2}}{\partial v \partial \partial u} K(u, v, s, t)
$$

$$
\begin{gathered}
\frac{\partial}{\partial x} \dot{\phi}(x, s, t) \equiv A(x, s, t)=\lim _{u \rightarrow x^{-}} \frac{\partial}{\partial u} \phi(u, s, t), \\
\frac{\partial}{\partial y} \psi(y, s, t) \equiv B(y, s, t)=\lim _{v \rightarrow y^{-}} \frac{\partial}{\partial v} \psi(v, s, t),
\end{gathered}
$$

and assume that there exists an integrable function $M(s, t)$ such that for all $(s, t) \in I^{2}$

$$
\left.\begin{array}{l}
\sup _{(x y) \in I^{2}}|H(x, y, s, t)| \\
\operatorname{var}_{\langle x y) \in I^{2}} H(x, y, s, t) \\
\operatorname{var}_{x \in I} H(x, 1, s, t) \\
\operatorname{var}_{y \in I} H(1, y, s, t) \\
\operatorname{var}_{x \in I} A(x, s, t) \\
\operatorname{var}_{y \in I} B(y, s, t)
\end{array}\right\} \leqq M(s, t) .
$$

Also assume that

$$
\begin{gathered}
|A(x, s, t)|,|B(y, s, t)| \leqq \beta, \text { a constant, and } \\
D(K) \neq 0 .
\end{gathered}
$$

Then for any Yeh-Wiener measurable functional $F(f)$, we have under the Fredholm transformation (1.1)

$$
\int_{C_{Y}} F(g) d_{Y} g \stackrel{*}{=}|D(K)| \int_{C_{Y}} F(T f) \cdot \exp \{-\Phi(f)\} d_{Y} f,
$$

where

$$
\begin{aligned}
\Phi(f)= & \int_{I^{2}}\left[\frac{\partial^{2}}{\partial y \partial x} \int_{I^{2}} K(x, y, s, t) f(s, t) d s d t\right]^{2} d x d y \\
& +2 \int_{I^{2}}\left[\frac{\partial^{2}}{\partial y \partial x} \int_{I^{2}} K(x, y, s, t) f(s, t) d s d t\right] d^{*} f(x, y),
\end{aligned}
$$

and “吕” indicates the existence of one side implies that of the other and the equality.

Theorem II. Let $h(x, y) \in L^{2}$ on $I^{2}, K(x, y, s, t)$ and $F(f)$ as in Theorem I. Then under the (nonlinear) transformation

$$
\begin{aligned}
L: f(x, y) \rightarrow g(x, y)= & f(x, y)+f_{0}(x, y) \\
& +\int_{1^{2}} K(x, y, x, s, t) f(s, t) d s d t,
\end{aligned}
$$


where $f_{0}(x, y)=\int_{0}^{y} \int_{0}^{x} h(s, t) d s d t$, we have

$$
\int_{C_{Y}} F(g) d_{Y} g \stackrel{*}{=}|D(K)| \int_{C_{Y}} F(L f) \cdot \exp \{-\Psi(f)\} d_{Y} f,
$$

where

$$
\begin{aligned}
\Psi(f)= & \int_{I^{2}}\left[h(x, y)+\frac{\partial^{2}}{\partial y \partial x} \int_{I^{2}} K(x, y, s, t) f(s, t) d s d t\right]^{2} d x d y \\
& +2 \int_{I^{2}}\left[h(x, y)+\frac{\partial^{2}}{\partial y \partial x} \int_{I^{2}} K(x, y, s, t) f(s, t) d s d t\right] d^{*} f(x, y) .
\end{aligned}
$$

3. Definitions. Cor responding to each continuous function $f(x, y)$ on $I^{2}$, the $\left(n^{\text {th }}\right)$ quasi-polyhedric function $f_{(n)}(x, y)$ of $f(x, y)$ is defined to be

$$
f_{(n)}(x, y)=a_{i j} x y+b_{i j} x+c_{i j} y+d_{i j}
$$

on each square $Q_{i j} \equiv[(i-1) / n, i / n] \times[(j-1) / n, j / n], i, j=1,2, \cdots, n$, where $a_{i j}, b_{i j}, c_{i j}$, and $d_{i j}$ are so chosen that $f_{(n)}(x, y)$ and $f(x, y)$ agree at the vertices $(i / n, j / n),(i / n,(j-1) / n),((i-1) / n, j / n)$ and $((i-1) / n$, $(j-1) / n)$.

REMARKs. (i). Since the function $f_{(n)}(x, y)$ is linear horizontally and vertically in each square $Q_{i j}$, we see that $f_{(n)}(x, y)$ is continuous on $I^{2}$. Furthermore the sequence $\left\{f_{(n)}(x, y)\right\}$ converges to $f(x, y)$ uniformly on $I^{2}$ as $n \rightarrow \infty$. Evaluating $a_{i j}, b_{i j}, c_{i j}$, and $d_{i j}$ explicitly, and then combining terms, we have on each square $Q_{i j}$,

$$
\begin{aligned}
f_{(n)}(x, y)= & f\left(\frac{i}{n}, \frac{j}{n}\right)\left[n^{2} x y-n(j-1) x-n(i-1) y+(i-1)(j-1)\right] \\
& +f\left(\frac{i-1}{n}, \frac{j}{n}\right)\left[-n^{2} x y+n(j-1) x+n i y-i(j-1)\right] \\
& +f\left(\frac{i}{n}, \frac{j-1}{n}\right)\left[-n^{2} x y+n j x+n(i-1) y-j(i-1)\right] \\
& +f\left(\frac{i-1}{n}, \frac{j-1}{n}\right)\left[n^{2} x y-n j x-n i y+i j\right] .
\end{aligned}
$$

(ii) If $K(x, y, s, t)$ is continuous on $I^{4}$, then for each $(s, t) \in I^{2}$ we can think of $K(x, y, s, t)$ as a function in $x, y$ and so we have the $\left(n^{\text {th }}\right)$ quasi-polyhedric function $K_{(n)}(x, y, s, t)$ in $x, y$, namely for $(x, y) \in$ $Q_{i j} ; i, j=1,2, \cdots, n$, we have 


$$
\begin{aligned}
& K_{(n)}(x, y, s, t) \\
&= K\left(\frac{i}{n}, \frac{j}{n}, s, t\right)\left[n^{2} x y-n(j-1) x-n(i-1) y+(i-1)(j-1)\right] \\
&+K\left(\frac{i-1}{n}, \frac{j}{n}, s, t\right)\left[-n^{2} x y+n(j-1) x+n i y-i(j-1)\right] \\
&+K\left(\frac{i}{n}, \frac{j-1}{n}, s, t\right)\left[-n^{2} x y+n j x+n(i-1) y-j(i-1)\right] \\
&+K\left(\frac{i-1}{n}, \frac{j-1}{n}, s, t\right)\left[n^{2} x y-n j x-n i y+i j\right] .
\end{aligned}
$$

We also see that $K_{(n)}(x, y, s, t) \rightrightarrows K(x, y, s, t)$ on $I^{4}$ as $n \rightarrow \infty$, provided that $K(x, y, s, t)$ is continuous on $I^{4}$. Here “ $\rightrightarrows$ " indicates uniform convergence.

For each $\varepsilon>0$ let

$$
\begin{aligned}
& \Omega_{\varepsilon}(s)=\operatorname{sgn} s-s / \varepsilon \quad \text { if } \quad|s| \leqq \varepsilon \\
& =0 \quad \text { if }|s|>\varepsilon \text {. }
\end{aligned}
$$

Let $K(x, y, s, t)$ and $\psi(y, s, t)$ be as in Theorem $\mathrm{I}$. Then the function defined by

$$
L_{\varepsilon}(x, y, s, t) \equiv K(x, y, s, t)+2^{-1}\left[\Omega_{\varepsilon}(s-x)-\Omega_{\varepsilon}\left(s^{+}\right)\right] \psi(y, s, t)
$$

is continuous in $x, s$. Now define

$$
J(x, s, t) \equiv L_{\varepsilon}\left(x, t^{+}, s, t\right)-L_{\varepsilon}\left(x, t^{-}, s, t\right),
$$

and

$$
\begin{aligned}
K_{\varepsilon}(x, y, s, t) \equiv & L_{\varepsilon}(x, y, s, t)+2^{-1}\left[\Omega_{\varepsilon}(t-y)-\Omega_{\varepsilon}\left(t^{+}\right)\right] J(x, s, t) \\
= & K(x, y, s, t)+2^{-1}\left[\Omega_{\varepsilon}(s-x) \Omega_{\varepsilon}\left(s^{+}\right)\right] \psi(y, s, t) \\
& +2^{-1}\left[\Omega_{\varepsilon}(t-y)-\Omega_{\varepsilon}\left(t^{+}\right)\right] \phi(x, s, t) \\
& +4^{-1}\left[\Omega_{\varepsilon}(s-x)-\Omega_{\varepsilon}\left(s^{+}\right)\right]\left[\Omega_{\varepsilon}(t-y)-\Omega_{\varepsilon}\left(t^{+}\right)\right]\left(K_{1}+K_{2}\right. \\
& \left.-K_{3}-K_{4}\right)(s, t, s, t) .
\end{aligned}
$$

Then $K_{s}(x, y, s, t)$ is continuous on $I^{4}$, and $K_{\varepsilon}(0, y, s, t)=K_{\varepsilon}(x, 0, s, t)$ $\equiv 0$. Furthermore $K_{\varepsilon}(x, y, s, t)$ is uniformly bounded in $\varepsilon, x, y, s, t$, and $\lim _{s \rightarrow 0+} K_{\varepsilon}(x, y, s, t)=K(x, y, s, t)$. Now, define

$$
\begin{aligned}
C_{\varepsilon}(x, s) & =\varepsilon / 2 & & \text { if } \\
& =0 & & \text { otherwise }
\end{aligned}
$$

Then from (3.7), (3.4), (3.8), and (2.3) it follows 


$$
\begin{aligned}
H_{\varepsilon}(x, y, s, t) \equiv & \frac{\partial^{2}}{\partial y \partial x} K_{s}(x, y, s, t) \\
= & H(x, y, s, t)+C_{\varepsilon}(x, s) B(y, s, t)+C_{\varepsilon}(y, t) A(x, s, t) \\
& +C_{\varepsilon}(x, s) \cdot C_{s}(y, t) \cdot\left(K_{1}+K_{2}-K_{3}-K_{4}\right)(s, t, s, t),
\end{aligned}
$$

with the understanding that whenever the partial derivatives are not defined at a point, then the value at the point to be the left-hand limit with respect to $x$ and $y$ (for the uniqueness sake) as in (2.3). Thus by using the mean-value property and the dominated convergence, we see that for almost all $(x, y)$ in $I^{2}$

$$
\begin{aligned}
\lim _{\varepsilon \rightarrow 0+} & \int_{I^{2}} H_{\varepsilon}(x, y, s, t) f(s, t) d s d t \\
= & \int_{L^{2}} H(x, y, s, t) f(s, t) d s d t+\int_{0}^{1} B(y, x, t) f(x, t) d t \\
& \quad+\int_{0}^{1} A(x, s, y) f(s, y) d s+\left(K_{1}+K_{2}-K_{3}-K_{4}\right)(x, y, x, y) \cdot f(x, y) .
\end{aligned}
$$

But the right hand side of (3.10) is exactly equal to

$$
\left(\partial^{2} / \partial y \partial x\right) \int_{I^{2}} K(x, y, s, t) f(s, t) d s d t .
$$

Therefore for a.a. $(x, y)$ in $I^{2}$

$$
\begin{gathered}
\lim _{\varepsilon \rightarrow 0+} \int_{I^{2}} \frac{\partial^{2}}{\partial y \partial x} K_{\varepsilon}(x, y, s, t) f(s, t) d s d t \\
\quad=\frac{\partial^{2}}{\partial y \partial x} \int_{I^{2}} K(x, y, s, t) f(s, t) d s d t .
\end{gathered}
$$

\section{Some Preliminary Lemmas.}

Lemma 1. Let $K_{(n)}(x, y, s, t)$ be the $n$-th polyhedric function in $(x, y)$ with the understanding that $K_{(n)}(x, y, s, t)=0$ for all $(x, y, s, t) \notin$ $I^{4}$. For $i, j, p, q=1,2, \cdots, n$ let

$$
A_{i j p q}^{(n)} \equiv \int_{(q-1) / n}^{(q+1) / n} \int_{(p-1) / n}^{(p+1) / n} K_{(n)}\left(\frac{i}{n}, \frac{j}{n}, s, t\right)(1-|n s-p|)(1-|n t-q|) d s d t .
$$

Then for any $f \in C_{Y}$ we obtain:

$$
\begin{aligned}
& \text { (i) } \int_{I^{2}} K_{(n)}\left(\frac{i}{n}, \frac{j}{n}, s, t\right) f_{(n)}(s, t) d s d t=\sum_{p, q=1}^{n} A_{i j p q}^{(n)} f\left(\frac{p}{n}, \frac{q}{n}\right), \\
& \text { (ii) } \int_{I^{2}} \frac{\partial^{2}}{\partial y \partial x} K_{(n)}(x, y, s, t) f_{(n)}(s, t) d s d t=n^{2} \sum_{p, q=1}^{n} B_{i j p q}^{(n)} f\left(\frac{p}{n}, \frac{q}{n}\right)
\end{aligned}
$$


for $(x, y) \in\left(\frac{i-1}{n}, \frac{i}{n}\right) \times\left(\frac{j-1}{n}, \frac{j}{n}\right)$, where

$$
B_{i j p q}^{(n)} \equiv A_{i j p q}^{(n)}-A_{i-1, j p q}^{(n)}-A_{i, j-1, p q}^{(n)}+A_{i-1, j-1 p q}^{(n)},
$$

(iii)

$$
\begin{gathered}
\int_{I^{2}}\left[\int_{I^{2}} \frac{\hat{\partial}^{2}}{\partial y \partial x} K_{(n)}(x, y, s, t) f_{(n)}(s, t) d s d t\right]^{2} d x d y \\
=n^{2} \sum_{i, j=1}^{n}\left[\sum_{p, q=1}^{n} B_{i s p q}^{(n)} f\left(\frac{p}{n}, \frac{q}{n}\right)\right]^{2},
\end{gathered}
$$

(iv)

$$
\begin{gathered}
\int_{l^{2}}\left[\int_{1^{2}} \frac{\partial^{2}}{\partial y \partial x} K_{(n)}(x, y, s, t) f_{(n)}(s, t) d s d t\right] d f_{(n)}(x, y) \\
=n^{2} \sum_{i, j=1}^{n}\left[\sum_{p, q=1}^{n} B_{i j p q}^{(n)} f\left(\frac{p}{n}, \frac{q}{n}\right)\right] \cdot \Delta_{i j} f_{(n)}
\end{gathered}
$$

where

$$
\begin{aligned}
\Delta_{i j} f_{(n)} \equiv & f\left(\frac{i}{n}, \frac{j}{n}\right)-f\left(\frac{i-1}{n}, \frac{j}{n}\right)-f\left(\frac{i}{n}, \frac{j-1}{n}\right) \\
& +f\left(\frac{i-1}{n}, \frac{j-1}{n}\right) .
\end{aligned}
$$

The proof of this lemma is similar to that of corresponding results in [1]. Next, we consider a transformation of $C_{Y}$ to $C_{Y}$ :

$$
T: g(x, y)=f_{(n)}(x, y)+\int_{I^{2}} K_{(n)}(x, y, s, t) f_{(n)}(s, t) d s d t .
$$

Then by (i) of Lemma 1 and the fact that $f_{(n)}(i / n, j / n)=f(i / n, j / n)$ at each $i$ and $j$, we have

$$
T: g\left(\frac{i}{n}, \frac{j}{n}\right)=f\left(\frac{i}{n}, \frac{j}{n}\right)+\sum_{p, q=1}^{n} A_{i j p q}^{(n)} f\left(\frac{p}{n}, \frac{q}{n}\right) ; \quad i, j=1,2, \cdots, n .
$$

The determinant $\Delta\left(K_{(n)}\right)$ of this transformation is given by

$$
\Delta\left(K_{(n)}\right) \equiv \operatorname{det}\left(A_{I P}^{*}+\delta_{I P}\right)_{I, P}=1,2, \cdots, n^{2}
$$

where $A_{I P}^{*}=A_{i j p q}^{(n)}$ with $I \equiv(i-1) n+j, P \equiv(p-1) n+q, 1 \leqq i, j, p, q \leqq$ $n$.

LEMMA 2. Let $F(f)$ be a Yeh-Wiener measurable functional which depends only on the $n^{2}$ values of $f(x, y)$ at $(x, y)=(i / n, j / n) ; i, j=$ $1,2, \cdots, n$. Let the $n^{\text {th }}$ quasi-polyhedric function in $x, y, K_{(n)}(x, y, s, t)$, satisfy that $K_{(n)}(x, y, s, t)=0$ if $x=0$ or $y=0$, and that $\Delta\left(K_{(n)}\right) \neq 0$. Then 


$$
\begin{aligned}
\int_{C_{Y}} F(g) d_{I^{\prime}} g \stackrel{*}{=} & \left|\Delta\left(K_{(n)}\right)\right| \int_{C_{Y}} F\left[f_{(n)}+\int_{I^{2}} K_{(n)}(\cdot, \cdot, s, t) f_{(n)}(s, t) d s d t\right] \\
& \exp \left\{-\int_{I^{2}}\left[\int_{I^{2}} \frac{\partial^{2}}{\partial y \partial x} K_{(n)}(x, y, s, t) f_{(n)}(s, t) d s d t\right]^{2} d x d y\right. \\
& \left.-2 \int_{I^{2}}\left[\int_{I^{2}} \frac{\partial^{2}}{\partial y \partial x} K_{(n)}(x, y, s, t) f_{(n)}(s, t) d s d t\right] d f_{(n)}(x, y)\right\} d_{Y} f .
\end{aligned}
$$

In view of Lemma 1 and a Yeh-Wiener integral formula (see Theorem I, [11]) the proof is word for word identical to that of the corresponding lemma in [1].

Lemma 3. If $K(x, y, s, t)$ is continuous on $I^{4}$, and if the Fredholm determinant $D(K) \neq 0$, then

$$
\lim _{n \rightarrow \infty} \Delta\left(K_{(n)}\right)=D(K) .
$$

Proof. Using (4.3), we may expand $\Delta\left(K_{(n)}\right)$ as:

$$
\begin{aligned}
& \Delta\left(K_{(n)}\right)=\operatorname{det}\left(A_{I P}^{*}+\delta_{I P}\right)_{I, P=12,}, \cdots, n^{2} \\
& =1+\sum_{P=1}^{n^{2}} A_{P P}^{*}+\frac{1}{2 !} \sum_{P_{1}, P_{2}=1}^{n^{2}} \operatorname{det}\left(A_{P_{i} P_{j}}^{*}\right)_{i, j=1,2} \\
& +\cdots+\frac{1}{\left(n^{2}\right) !} \sum_{P_{1}, \cdots, P^{2}{ }^{2}=1}^{n^{2}} \operatorname{det}\left(A_{P_{i} P_{j}}^{*}\right)_{i, j=1}, \cdots n^{2} \\
& =1+\sum_{p, q=1}^{n} A_{p q p q}^{(n)}+\frac{1}{2 !} \sum_{p_{1}, q_{1}, p_{2}, q_{2}=1}^{n} \operatorname{det}\left(A_{p_{i} q_{i} p_{j} q_{j}}^{(n)}\right)_{i j=12} \\
& +\cdots+\frac{1}{\left(n^{2}\right) !} \sum_{p_{1}, q_{1}, p_{2}, q_{2}, \cdots, p_{n}, q_{n^{2}}=1}^{n} \operatorname{det}\left(A_{p_{i} q_{i} p_{j} q_{j}}^{(n)}\right)_{i j=12} \cdots n^{2} \cdot
\end{aligned}
$$

Let $M$ be the bound for $K$ on $I^{4}$. Then by (4.1) we have $\left|n^{2} A_{i j p q}^{(n)}\right| \leqq$ $M$ uniformly in $n, i, j, p, q$. Thus by Hadamard's inequality it follows:

$$
\left|\operatorname{det}\left(A_{p_{i} q_{i} p_{j} q_{j}}^{(n)}\right)_{i, j=1}, \cdots, N\right| \leqq\left(M / n^{2}\right)^{N} N^{N / 2} .
$$

Upon using (4.5) in (4.4), we conclude that $\left|\Delta\left(K_{(n)}\right)\right|$ is uniformly bounded by the convergent series $1+\sum_{N=1}^{\infty} M^{N} N^{N / 2} / N$ !, and for each $N$

$$
\begin{aligned}
& \lim _{n \rightarrow \infty} \sum_{p_{1}, q_{1}, \cdots, p_{N}, q_{N}=1}^{n} \operatorname{det}\left(A_{p_{i} q_{i} p_{j} q_{j}}^{(n)}\right)_{i}{ }_{j=1}, \cdots, N
\end{aligned}
$$

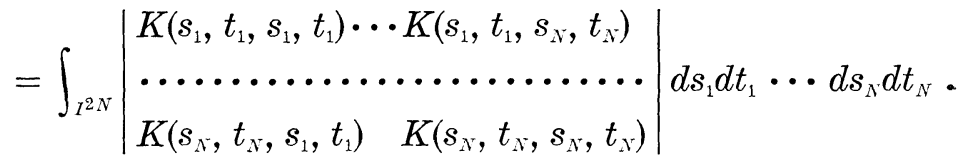

Hence the conclusion follows.

Similarly it follows: 
LEMma 4. Let $K(x, y, s, t)$ be a bounded integrable function with $D(K) \neq 0$ and let $\left\{K_{\lambda}(x, y, s, t)\right\}$ be a set of Borel measurable functions which are uniformly bounded in $\lambda, x, y, s, t$, and let $\lim _{\lambda \rightarrow 0^{+}} K_{\lambda}(x, y, s, t)=$ $K(x, y, s, t)$. Then $\lim _{\lambda \rightarrow 0^{+}} D\left(K_{\lambda}\right)=D(K)$.

Lemma 5. For each $(s, t) \in I^{2}$ let $H(x, y, s, t)$ satisfy that

$$
\sup _{(x, y) \in I^{2}}|H(x, y, s, t)|, \operatorname{var}_{(x, y) \in I^{2}} H(x, y, s, t), \operatorname{var}_{x \in I} H(x, 1, s, t), \operatorname{var}_{y \in I} H(1, y, s, t)
$$

are all dominated by an integrable function $M(s, t)$. Then

$$
\begin{aligned}
& \int_{I^{2}}\left[\int_{I^{2}} H(x, y, s, t) f(s, t) d s d t\right] d f(x, y) \\
& \quad=\int_{I^{2}} f(s, t)\left[\int_{I^{2}} H(x, y, s, t) d f(x, y) d s d t .\right.
\end{aligned}
$$

Proof. Let " $\|\cdot\|$ " denote the supremum of the absolute value. Then from the fact that

$$
\left.\begin{array}{l}
\operatorname{var}_{(x, y) \in I^{2}} \int_{I^{2}} H(x, y, s, t) f(s, t) d s d t \\
\operatorname{var}_{y \in I} \int_{I^{2}} H(x, 1, s, t,) f(s, t) d s d t \\
\operatorname{var}_{y \in I} \int_{I^{2}} H(1, y, s, t) f(s, t) d s d t
\end{array}\right\} \leqq\|f\| \int_{I^{2}} M(s, t) d s d t<\infty
$$

the left member of (4.6) exists as an ordinary $R-S$ integral. Hence for the net: $x_{i}=i / n, y_{j}=j / n ; i, j=1,2, \cdots, n$, and $x_{i-1} \leqq x_{i}^{*} \leqq x_{i}$, $y_{j-1} \leqq y_{j}^{*} \leqq y_{j}$, we have with $\Delta_{i j} f(x, y)=f\left(x_{i}, y_{j}\right)-f\left(x_{i-1}, y_{j}\right)-$ $f\left(x_{i}, y_{j-1}\right)+f\left(x_{i-1}, y_{j-1}\right)$,

$$
\begin{aligned}
& \int_{I^{2}}\left[\int_{I^{2}} H(x, y, s, t) f(s, t) d s d t\right] d f(x, y) \\
& \quad=\lim _{n \rightarrow \infty} \sum_{i, j=1}^{n}\left[\int_{I} H\left(x_{i}^{*}, y_{j}^{*}, s, t\right) f(s, t) d s d t\right] \Delta_{\imath j} f(x, y) \\
& \quad=\lim _{n \rightarrow \infty} \int_{2} f(s, t)\left[\sum_{i, j=1}^{n} H\left(x_{i}^{*}, y_{j}^{*}, s, t\right) \Delta_{i j} f(x, y)\right] d s d t .
\end{aligned}
$$

But

$$
\begin{array}{rl}
\mid \sum_{i, j=1}^{n} & H\left(x_{i}^{*}, y_{j}^{*}, s, t\right) \Delta_{i j} f(x, y) \mid \\
\leqq & \|f\|\left[\operatorname{var}_{(x, y) \in 1^{2}} H(x, y, s, t)+\operatorname{var}_{x \in I} H(x, 1, s, t)+\operatorname{var}_{y \in I} H(1, y, s, t)\right. \\
& +|H(1,1, s, t)|] \\
\leqq & 4\|f\| M(s, t),
\end{array}
$$

and since $M(s, t)$ is finite a.e. we see that for a.a. $(s, t) \in I^{2}$ 


$$
\lim _{n \rightarrow \infty} \sum_{i, j=1}^{n} H\left(x_{i}^{*}, y_{j}^{*}, s, t\right) \Delta_{i j} f(x, y)=\int_{I^{2}} H(x, y, s, t) d f(x, y) .
$$

Thus (4.6) follows from this, (4.8) and (4.9) by dominated convergence.

Lemma 6. Let $H(x, y, s, t)$ be as in Lemma 5, and let

$$
H^{n}(x, y, s, t) \equiv n^{2} \int_{(q-1) / n}^{q / n} \int_{(p-1) / n}^{p / n} H(u, v, s, t) d u d v
$$

for $(x, y) \in\left(\frac{p-1}{n}, \frac{p}{n}\right] \times\left(\frac{q-1}{n}, \frac{q}{n}\right], p, q=1,2, \cdots, n$.

Then for every $f(x, y) \in C_{Y}$

$$
\begin{aligned}
\lim _{n \rightarrow \infty} \int_{I^{2}}\left[\int_{I^{2}} H(x, y, s, t) f_{(n)}(s, t) d s d t\right]^{2} d x d y \\
=\int_{I^{2}}\left[\int_{I^{2}} H(x, y, s, t) f(s, t) d s d t\right]^{2} d x d y,
\end{aligned}
$$

$$
\begin{gathered}
\left|\int_{I^{2}}\left[\int_{I^{2}} H^{n}(x, y, s, t) f_{(n)}(s, t) d s d t\right]^{2} d x d y\right| \leqq\left(\|f\| \int_{I^{2}} M(s, t) d s d t\right)^{2} \\
\lim _{n \rightarrow \infty} \int_{I^{2}}\left[\int_{I^{2}} H^{n}(x, y, s, t) f_{(n)}(s, t) d s d t\right] d f_{(n)}(x, y) \\
=\int_{I^{2}}\left[\int_{I^{2}} H(x, y, s, t) f(s, t) d s d t\right] d f(x, y) .
\end{gathered}
$$

Proof. (4.11) and (4.12) are immediate. By (4.7) the function $\int_{I^{2}} H(x, y, s, t) f(s, t) d s d t$ is of B.V. Now

$$
\begin{aligned}
& \int_{I^{2}}\left[\int_{I^{2}} H^{n}(x, y, s, t) f(s, t) d s d t\right] d f(x, y) \\
& \quad=\sum_{p, q=1}^{n} \int_{I^{2}}\left[n^{2} \int_{(p-1) / n}^{q / n} \int_{(q-1) / n}^{p / n} H(u, v, s, t) d u, d v\right] f(s, t) d s d t \cdot \Delta_{p_{q}} f,
\end{aligned}
$$

where $\Delta_{p, q} f=f\left(\frac{p}{n}, \frac{q}{n}\right)-f\left(\frac{p-1}{n}, \frac{q}{n}\right)-f\left(\frac{p}{n}, \frac{q-1}{n}\right)+f\left(\frac{p-1}{n}, \frac{q-1}{n}\right)$. Since

$$
\int_{2}\left[n^{2} \int_{(q-1) / n}^{q / n} \int_{(p-1) / n}^{p / n} H(u, v, s, t) d u d v\right] f(s, t) d s d t
$$

is the average value of $\int_{I^{2}} H(x, y, s, t) f(s, t) d s d t$ in the square $((q-1) / n, q / n] \times((q-1) / n, p / n]$, the existence of the $R-S$ integral implies 


$$
\begin{aligned}
\lim _{n \rightarrow \infty} \int_{I^{2}}\left[\int_{I^{2}} H^{n}(x, y, s, t) f(s, t) d s d t\right] d f(x, y) \\
=\int_{I^{2}}\left[\int_{I^{2}} H(x, y, s, t) f(s, t) d s d t\right] d f(x, y) .
\end{aligned}
$$

Now,

$$
\begin{aligned}
& \operatorname{var}_{(x, y) \in I^{2}} H^{n}(x, y, s, t) \leqq \operatorname{var}_{(x, y) \in I^{2}} H(x, y, s, t) \leqq M(s, t) \\
& \operatorname{var}_{x \in I} H^{n}(x, 1, s, t) \leqq \operatorname{var}_{x \in I} H(x, 1, s, t) \leqq M(s, t) \\
& \operatorname{var}_{y \in I} H^{n}(1, y, s, t) \leqq \operatorname{var}_{y \in I} H(1, y, s, t) \leqq M(s, t) \\
& \left|H^{n}(1,1, s, t)\right| \leqq \sup _{(x, y) \in I^{2}}|H(x, y, s, t)| \leqq M(s, t) .
\end{aligned}
$$

Seeing the fact that $H^{n}(x, y, s, t)$ is constant in $x, y$ in each square $\left(\frac{p-1}{n}, \frac{p}{n}\right]\left(\frac{q-1}{n}, \frac{q}{n}\right]$ and that $f_{(n)}(x, y)$ and $f(x, y)$ agree on the vertices of this square, we can write

$$
\begin{gathered}
\int_{I^{2}}\left[\int_{I^{2}} H^{n}(x, y, s, t) f_{(n)}(s, t) d s d t\right] d f_{(n)}(x, y) \\
\quad=\sum_{p, q=1}^{n} \int_{I^{2}} H^{n}\left(\frac{p}{n}, \frac{q}{n}, s, t\right) f_{(n)}(s, t) d s d t \cdot \Delta_{p q} f \\
\quad=\int_{I^{2}} f_{(n)}(s, t)\left[\sum_{p, q=1}^{n} H^{n}\left(\frac{p}{n}, \frac{q}{n}, s, t\right) \Delta_{p q} f\right] d s d t .
\end{gathered}
$$

Therefore

$$
\begin{aligned}
& \mid \int_{I^{2}}\left[\int_{I^{2}} H^{n}(x, y, s, t) f(s, t) d s d t\right] d f(x, y) \\
& \quad-\int_{I^{2}}\left[\int_{I^{2}} H^{n}(x, y, s, t) f_{(n)}(s, t) d s d t\right] d f_{(n)}(x, y) \mid \\
& \quad=\left|\int_{I^{2}}\left[f(s, t)-f_{(n)}(s, t)\right]\left[\sum_{p, q=1}^{n} H^{n}\left(\frac{p}{n}, \frac{q}{n}, s, t\right) \cdot \Delta_{p q} f\right] d s d t\right| \\
& \quad \leqq 4\left\|f-f_{n}\right\|\|f\| \int_{I^{2}} M(s, t) d s d t,
\end{aligned}
$$

thus obtaining

$$
\begin{aligned}
\lim _{n \rightarrow \infty}[ & {\left[\int_{2} H_{I^{2}} H^{n}(x, y, s, t) f_{(n)}(s, t) d s d t\right] d f_{(n)}(x, y) } \\
& =\lim _{n \rightarrow \infty} \int_{I^{2}}\left[\int_{I^{2}} H^{n}(x, y, s, t) f(s, t) d s d t\right] d f(x, y) .
\end{aligned}
$$

Hence (4.13) follows from (4.14) and (4.16).

Corollary. Let $K(x, y, s, t)$ be as in Theorem $I, K_{\varepsilon}(x, y, s, t)$ the 
corresponding function defined by (3.7), and $K_{\varepsilon,(n)}(x, y, s, t)$ the $n^{\text {th }}$ quasi-polyhedric function of $K_{\varepsilon}$ in $x, y$. Then

$$
\begin{gathered}
\lim _{n \rightarrow \infty} \int_{I^{2}}\left[\int_{I^{2}} \frac{\partial^{2}}{\partial y \partial x} K_{\varepsilon,(n)}(x, y, s, t) f_{(n)}(s, t) d s d t\right]^{2} d x d y \\
=\int_{I^{2}}\left[\int_{I^{2}} \frac{\partial^{2}}{\partial y \partial x} K_{\varepsilon}(x, y, s, t) f(s, t) d s d t\right]^{2} d x d y, \\
\lim _{n \rightarrow \infty} \int_{I^{2}}\left[\int_{I^{2}} \frac{\partial^{2}}{\partial y \partial x} K_{\varepsilon,(n)}(x, y, s, t) f_{(n)}(s, t) d s d t\right] d f_{(n)}(x, y) \\
=\int_{I^{2}}\left[\int_{I^{2}} \frac{\partial^{2}}{\partial y \partial x} K_{\varepsilon}(x, y, s, t) f(s, t) d s d t\right] d f(x, y) .
\end{gathered}
$$

Proof. In view of (3.9), (3.8), and (2.4) we obtain

$$
\begin{aligned}
\sup _{(x, y) \in I^{2}}\left|H_{\varepsilon}(x, y, s, t)\right| \leqq & M(s, t)+\frac{\beta}{\varepsilon}+\frac{1}{4 \varepsilon^{2}} \mid\left(K_{1}+K_{2}-K_{3}\right. \\
& \left.-K_{4}\right)(s, t, s, t) \mid, \\
\operatorname{var}_{(x, y) \in I^{2}} H_{\varepsilon}(x, y, s, t) \leqq & M(s, t)+\frac{2}{\varepsilon} M(s, t)+\frac{1}{\varepsilon^{2}} \mid\left(K_{1}+K_{2}-K_{3}\right. \\
& \left.-K_{4}\right)(s, t, s, t) \mid,
\end{aligned}
$$

$$
\begin{aligned}
\operatorname{var}_{x \in I} H_{\varepsilon}(x, 1, s, t) \leqq & M(s, t)+\frac{1}{2 \varepsilon}[2 \beta+M(s, t)]+\frac{1}{2 \varepsilon^{2}} \mid\left(K_{1}+K_{2}\right. \\
& \left.-K_{3}-K_{4}\right)(s, t, s, t) \mid, \\
\operatorname{var}_{y \in I} H_{\varepsilon}(1, y, s, t) \leqq & M(s, t)+\frac{1}{2 \varepsilon}[2 \beta+M(s, t)]+\frac{1}{2 \varepsilon^{2}} \mid\left(K_{1}+K_{2}\right. \\
& \left.-K_{3}-K_{4}\right)(s, t, s, t) \mid .
\end{aligned}
$$

Let $M_{\varepsilon}(s, t)$ be the sum of the four right members of (4.19). From (3.3) it is obvious that

$$
\frac{\partial^{2}}{\partial x \partial y} K_{\varepsilon(n)}(x, y, s, t)=n^{2} \int_{(q-1) / n}^{q / n} \int_{(p-1) / n}^{p / n} \frac{\partial^{2}}{\partial v \partial u} K_{\varepsilon}(u, v, s, t) d u d v
$$

for $(p-1) / n<x \leqq p / n,(q-1) / n<y \leqq q / n ; p, q=1, \cdots, n$. Hence from (4.10), (4.20), and the fact that $\left(\partial^{2} / \partial v \partial u\right) K_{\varepsilon}(u, v, s, t) \equiv H_{\varepsilon}(u, v, s, t)$, we see that

$$
\frac{\partial^{2}}{\partial y \partial x} H_{\varepsilon \cdot(n)}(x, y, s, t) \equiv H_{s}^{n}(x, y, s, t) .
$$

Thus the conclusions follow directly from Lemma 6 • 
5. Some discussions on resolvent kernels. Let us denote the resolvent kernel of $K(x, y, s, t)$ in the Fredholm transformation (1.1) by $K^{*}(x, y, s, t)$, i.e., if

$$
g(x, y)=f(x, y)+\int_{I^{2}} K(x, y, s, t) f(s, t) d s d t,
$$

then

$$
f(x, y)=g(x, y)+\int_{I^{2}} K^{*}(x, y, s, t) g(s, t) d s d t .
$$

Let $K(x, y, s, t)$ be a bounded $L^{2}$-kernel on $I^{4}$ with $D(K) \neq 0$, and set

$$
\mathscr{D}(x, y, s, t) \equiv K^{*}(x, y, s, t) \cdot D(K) .
$$

Then, using the familiar results for resolvent kernels for Fredholm integral equations with $\lambda=-1$ (see [8], pp. 66-75), we can establish

$$
\begin{gathered}
K(x, y, s, t)+K^{*}(x, y, s, t)=-\int_{I^{2}} K(x, y, u, v) \cdot K^{*}(u, v, s, t) d u d v, \\
\mathscr{X}(x, y, s, t)=\sum_{n=0}^{\infty} C_{n}(x, y, s, t) / n !
\end{gathered}
$$

where $C_{0}(x, y, s, t)=-K(x, y, s, t)$, and other $C_{n}^{\prime} s$ are found successively by

$$
\begin{aligned}
& C_{n}(x, y, s, t)= J_{n}(K) \cdot K(x, y, s, t) \\
&-n \int_{I^{2}} K(x, y, u, v) C_{n-1}(u, v, s, t) d u d v, \\
& J_{n}(K)=-\int_{I^{2}} \stackrel{(n)}{\cdots} \int_{I^{2}}\left|\begin{array}{r}
K\left(x_{1}, y_{1}, x_{1}, y_{1}\right) \cdots K\left(x_{1}, y_{1}, x_{n}, y_{n}\right) \\
\cdots\left(x_{n}, y_{n}, x_{1}, y_{1}\right) \cdots K\left(x_{n}, y_{n}, x_{n}, y_{n}\right)
\end{array}\right| \\
& d x_{1} d y_{1} \cdots d x_{n} d y_{n} .
\end{aligned}
$$

By Hadamard's inequality it follows from (5.6) that

$$
\left|C_{n}(x, y, s, t)\right| \leqq\left.(n+1)^{(n+1) / 2}|| K\right|^{n+1} \text {. }
$$

Therefore the series in (5.5) is absolutely and uniformly convergent. If $K(x, y, s, t)$ satisfies the assumptions in Theorem $\mathrm{I}$, then

$$
\int_{T^{2}} K(x, y, u, v) C_{n}(u, v, s, t) d u d v
$$

is continuous on $I^{4}$ for $n=0,1,2, \cdots$, and hence from (5.6) we see that the jumps for $C_{n}(u, v, s, t)$ coincides with those for $K(x, y, s, t)$, and thus it takes average value at each jump, and so does $\sum_{n=0}^{\infty} C_{n}(x, y, s, t) / n$ ! by uniform convergence. By absolute convergence 
we may rearrange the terms in the series (5.5), and then use the uniform convergence to obtain

$$
\begin{aligned}
\mathscr{D}(x, y, s, t)= & \sum_{n=0}^{\infty} \frac{1}{n !}\left[J_{n} \cdot K(x, y, s, t)\right. \\
& \left.-n \int_{I^{2}} K(x, y, u, v) . C_{n-1}(u, v, s, t) d u d v\right] \\
= & C \cdot K(x, \mathrm{y}, s, t)-\int_{I^{2}} K(x, y, u, v) \cdot \mathscr{D}(u, v, s t) d u d v,
\end{aligned}
$$

where $C=\sum_{n=0}^{\infty} J_{n}(K) / n$ !. Corresponding to each $K \in(x, y, s, t)$, define $K_{(\varepsilon)}^{*}(x, y, s, t)$ by

$$
\begin{aligned}
K_{(\varepsilon)}^{*}(x, y, s, t)= & D(K) \mathscr{D}_{(\varepsilon)}(x, y, s, t) \\
= & D(K)\left[C \cdot K_{s}(x, y, s, t)\right. \\
& \left.-\int_{I^{2}} K_{s}(x, y, u, v) \cdot \mathscr{D}(u, v, s, t) d u d v\right] .
\end{aligned}
$$

Then $K_{(\varepsilon)}^{*}(x, y, s, t)$ is continuous on $I^{4}$, and since $\mathscr{D}(u, v, s, t)$ is bounded, we have that $K_{(\varepsilon)}^{*}(x, y, s, t)$ is uniformly bounded in $\varepsilon, x, y, s, t$, and from (3.9), (4.19), and (5.8) it follows that

$$
\begin{aligned}
H_{(\varepsilon)}^{*}(x, y, s, t) \equiv & \frac{\partial^{2}}{\partial y \partial x} K_{(\varepsilon)}^{*}(x, y, s, t) \\
= & D(K)\left[C \cdot H_{\varepsilon}(x, y, s, t)\right. \\
& \left.-\int_{T^{2}} H_{\varepsilon}(x, y, u, v) \cdot \mathscr{D}(u, v, s, t) d u d v\right],
\end{aligned}
$$

and $\sup _{(x y) \in I^{2}}\left|H_{(\varepsilon)}^{*}(x, y, s, t)\right|, \operatorname{var}_{(x, y) \in I^{2}} H_{(\varepsilon)}^{*}(x, y, s, t), \operatorname{var}_{x \in I} H_{(\varepsilon)}^{*}(x, 1, s, t)$, and $\operatorname{var}_{y \in I} H_{(\varepsilon)}^{*}(1, y, s, t)$ are all dominated by $|D(K)|(|C|+\|\mathscr{D}\|)$. $M_{\varepsilon}(s, t)$. Furthermore, by Lemma 4

$$
\lim _{\varepsilon \rightarrow 0+} D\left(K_{(\varepsilon)}^{*}\right)=D\left(K^{*}\right)=D^{-1}(K) .
$$

6. Additional lemmas. Utilizing (3.7), (3.9), (3.11), (5.8), and (5.9), we have the following

LEmma 7. If $K(x, y, s, t)$ satisfies the hypotheses of Theorem I, then

$$
\begin{aligned}
& \left|\int_{I^{2}}\left[K_{\varepsilon}(x, y, s, t)-K(x, y, s, t)\right] f(s, t) d s d t\right| \\
& \leqq \\
& \leqq \\
& \quad \int_{I^{2}}\left[\int_{I^{2}} \frac{\partial^{2}}{\partial y \partial x} K_{\varepsilon}(x, y, s, t) f(s, t) d s d t\right]^{2} d x d y
\end{aligned}
$$


$(6.2)$

$$
\leqq\|f\|^{2}\left[\int_{I^{2}} M(s, t) d s d t+2 \beta+4\|K\|\right]^{2},
$$

$$
\lim _{s \rightarrow 0^{+}} \int_{I^{2}}\left[\int_{I^{2}} \frac{\partial^{2}}{\partial y \partial x} K_{\varepsilon}(x, y, s, t) f(s, t) d s d t\right]^{2} d x d y
$$

$$
=\int_{I^{2}}\left[\frac{\partial^{2}}{\partial y \partial x} \int_{I^{2}} K(x, y, s, t) f(s, t) d s d t\right]^{2} d x d y,
$$

$$
\begin{gathered}
\left|\int_{I^{2}}\left[K_{(s)}^{*}(x, y, s, t)-K^{*}(x, y, s, t)\right] f(s, t) d s d t\right| \\
\leqq 4 \varepsilon(1+\varepsilon)\|f\|\|K\||D(K)| \cdot(|C|+\|\mathscr{D}\|), \\
\int_{I^{2}}\left[\int_{I^{2}} \frac{\partial^{2}}{\partial y \partial x} K_{(s)}^{*}(x, y, s, t) f(s, t) d s d t\right]^{2} d x d y \\
\leqq(\|f\| D(K))^{2}(|C|+\|\mathscr{D}\|)\left[\int_{I^{2}} M(s, t) d s d t+2 \beta+4\|K\|\right]^{2}, \\
\lim _{\varepsilon \rightarrow 0^{+}} \int_{I^{2}}\left[\int_{I^{2}} \frac{\partial^{2}}{\partial y \partial x} K_{(\varepsilon)}^{*}(x, y, s, t) f(s, t) d s d t\right]^{2} d x d y \\
=\int_{I^{2}}\left[\frac{\partial^{2}}{\partial y \partial x} K_{(\varepsilon)}^{*}(x, y, s, t) f(s, t) d s d t\right]^{2} d x d y .
\end{gathered}
$$

The following two lemmas are the key results:

Lemma 8. Let $K(x, y, s, t)$ be as in Theorem $I$. Then

$$
\int_{I^{2}}\left[\frac{\partial^{2}}{\partial y \partial x} \int_{I^{2}} K(x, y, s, t) f(s, t) d s d t\right] d^{*} f(x, y)
$$

defined as in (1.2) converges for a.a. $f$ in $C_{Y}$, and any two C.O.N. sets in the class lead to the same value for a.a. $f$ in $C_{Y}$. Furthermore, we have

$$
\begin{aligned}
& \underset{\varepsilon \rightarrow 0^{+}}{\operatorname{l.i.m}} \int_{I^{2}}\left[\int_{I^{2}} \frac{\partial^{2}}{\partial y \partial x} K_{\varepsilon}(x, y, s, t) f(s, t) d s d t\right] d f(x, y) \\
& \quad=\int_{I^{2}}\left[\frac{\partial^{2}}{\partial y \partial x} \int_{I^{2}} K(x, y, s, t) f(s, t) d s d t\right] d^{*} f(x, y) \text { on } C_{Y},
\end{aligned}
$$

where the mean convergence is in $L^{2}$-sense.

Proof. First we observe that

$$
\int_{I^{2}}\left[\frac{\partial^{2}}{\partial y \partial x} \int_{I^{2}} K(x, y, s, t) f(s, t) d s d t\right] d^{*} f(x, y)
$$




$$
\begin{aligned}
= & \int_{I^{2}}\left[\int_{I^{2}} \frac{\partial^{2}}{\partial y \partial x} K(x, y, s, t) f(s, t) d s d t\right] d^{*} f(x, y) \\
& +\int_{I^{2}}\left[\int_{0}^{1} B(y, x, t) f(x, t) d t\right] d^{*} f(x, y) \\
& +\int_{I^{2}}\left[\int_{0}^{1} A(x, s, y) f(s, y) d s\right] d^{*} f(x, y) \\
& +\int_{I^{2}}\left(K_{1}+K_{2}-K_{3}-K_{4}\right)(x, y, x, y) \cdot f(x, y) d^{*} f(x, y),
\end{aligned}
$$

and the existence and the consistency of each of last three expressions follow in the similar manner as in the generalized P.W.Z. integral $\int_{I^{2}} h f d^{*} f$. On account of (3.9) and Lemma 5 we may write

$$
\begin{aligned}
& \int_{I^{2}}\left[\int_{I^{2}} \frac{\partial^{2}}{\partial y \partial x} K_{\varepsilon}(x, y, s, t) f(s, t) d s d t\right] d f(x, y) \\
& =\int_{I^{2}}\left[\int_{I^{2}} \frac{\partial^{2}}{\partial y \partial x} K(x, y, s, t) f(s, t) d s d t\right] d f(x, y) \\
& \quad+\int_{I^{2}} f(s, t)\left[\int_{I^{2}} C_{\varepsilon}(x, s) B(y, s, t) d f(x, y)\right] d s d t \\
& \quad+\int_{I^{2}} f(s, t)\left[\int_{I^{2}} C_{\varepsilon}(y, t) A(x, s, t) d f(x, y)\right] d s d t \\
& \quad+\int_{I^{2}}\left(K_{1}+K_{2}-K_{3}-K_{4}\right)(s, t, s, t)\left[\int_{I^{2}} C_{s}(x, s) C_{\varepsilon}(y, t) d f(x, y)\right] d s d t .
\end{aligned}
$$

Obviously,

$$
\begin{aligned}
& \int_{I^{2}}\left[\int_{I^{2}} \frac{\partial^{2}}{\partial y \partial x} K(x, y, s, t) f(s, t) d s d t\right] d f(x, y) \\
& \quad=\int_{I^{2}}\left[\int_{I^{2}} \frac{\partial^{2}}{\partial y \partial x} K(x, y, s, t) f(s, t) d s d t\right] d^{*} f(x, y) \text { for a.a. } f \in C_{Y} .
\end{aligned}
$$

Thus to establish (6.6) we need only show that

$$
\begin{array}{r}
\underset{\varepsilon \rightarrow 0^{+}}{\operatorname{l.m.m}} \int_{I^{2}} f(s, t)\left[\int_{I^{\varepsilon}} C_{\epsilon}(x, s) B(y, s, t) d f(x, y)\right] d s d t \\
=\int_{I^{2}}\left[\int_{0}^{1} B(y, x, t) f(x, t) d t\right] d^{*} f(x, y) \text { on } C_{Y},
\end{array}
$$

and so on. To see this we observe that the Yeh-Wiener integral

$$
\begin{aligned}
\int_{C_{Y}} & \left\{\int_{I^{2}} f(s, t)\left[\int_{I^{2}} C_{s}(x, s) B(y, s, t) d f(x, y)\right] d s d t\right\}^{2} d_{Y} f \\
& =\frac{1}{4}\left\{\int_{I^{4}}\left[\int_{t}^{1} \int_{s}^{1} C_{s}\left(x, s^{\prime}\right) B\left(y, s^{\prime}, t^{\prime}\right) d s^{\prime} d t^{\prime}\right]^{2} d s d t d x d y\right.
\end{aligned}
$$




$$
\begin{aligned}
&+ {\left[\int_{I^{2}}\left(\int_{0}^{t} \int_{0}^{s} C_{\varepsilon}(x, s) B(y, s, t) d x d y\right) d s d t\right]^{2} } \\
&+ \int_{I^{4}}\left(\int_{0}^{t} \int_{0}^{s} C_{\varepsilon}\left(x, s^{\prime}\right) B\left(y, s^{\prime}, t^{\prime}\right) d x d y\right) \\
&\left.\times\left(\int_{0}^{t \prime} \int_{0}^{s \prime} C_{\varepsilon}(x, s) B(y, s, t) d x d y\right) d s d t d s^{\prime} d t^{\prime}\right\}, \\
& \int_{C_{Y}}\left\{\int_{I_{1}}\left[\int_{0}^{1} B(y, x, t) f(x, t) d t\right] d^{*} f(x, y)\right\}^{2} d_{Y} f \\
&=\frac{1}{4}\left\{\int_{I^{3}}\left[\int_{x}^{1}\left(\int_{t}^{1} B\left(y, x^{\prime}, t^{\prime}\right) d t^{\prime}\right)^{2} d x^{\prime}\right] d x d t d y\right. \\
&\left.+\frac{1}{4}\left[\int_{I^{2}}\left(\int_{y}^{1} B(y, x, t) d t\right) d x d y\right]^{2}\right\},
\end{aligned}
$$

and

$$
\begin{aligned}
& \int_{C_{Y}}\left\{\int_{I^{2}} f(s, t)\left[\int_{I^{2}} C_{s}(x, s) B(y, s, t) d f(x, y)\right] d s d t\right\} \\
& \cdot\left\{\int_{I^{2}}\left[\int_{0}^{1} B(y, x, t) f(x, t) d t\right] d^{*} f(x, y)\right\} d_{Y} f \\
& =\frac{1}{4}\left\{\int_{I^{3}}\left[\int_{I^{2}} B\left(y, x, t^{\prime}\right) \min (s, x) C_{\varepsilon}(x, s) B(y, s, t) d x d y\right] \min \left(t, t^{\prime}\right) d s d t d t^{\prime}\right. \\
& +\int_{I^{3}}\left(\int_{0}^{t} \int_{0}^{s} B\left(y^{\prime}, x^{\prime}, t_{\iota}\right)\left[\int_{0}^{t^{\prime}} \int_{0}^{x \prime} C_{\varepsilon}(x, s) B(y, s, t) d x d y\right] d x^{\prime} d y^{\prime}\right) d s d t d t^{\prime} \\
& \left.+\frac{1}{2} \int_{I^{4}}\left(\int_{0}^{t \prime} B\left(y^{\prime}, x^{\prime}, t^{\prime}\right)\left[\int_{0}^{t} \int_{0}^{s} C_{\varepsilon}(s, x) B(y, s, t) d x d y\right] d y^{\prime}\right) d x^{\prime} d s d t d t^{\prime}\right\}
\end{aligned}
$$

The techniques leading to the evaluation of above integrals can be found in [3] and [4]. Using these and then taking the limit as $\varepsilon \rightarrow 0^{+}$, we get

$$
\begin{aligned}
\lim _{\varepsilon \rightarrow 0^{+}} \int_{C_{Y}}\left\{\int_{I^{2}} f(s, t)\left[\int_{I^{2}} C_{\varepsilon}(x, s) B(y, s, t) d f(x, y)\right] d s d t\right. \\
\left.-\int_{I^{2}}\left[\int_{0}^{1} B(y, x, t) f(x, t) d t\right] d^{*} f(x, y)\right\}^{2} d_{Y} f=0,
\end{aligned}
$$

which is exactly the same as (6.9).

Lemma 8'. Let $K(x, y, s, t)$ satisfy the hypotheses of Theorem $I$. Then for its resolvent kernel $K^{*}$ we have

$$
\int_{I^{2}}\left[\frac{\partial^{2}}{\partial y \partial x} \int_{I^{2}} K^{*}(x, y, s, t) f(x, t) d s d t\right] d^{*} f(x, y)
$$

converges for almost all $f$ in $C_{Y}$ and is essentially independent of the particular choice of the C.O.N. set, and 


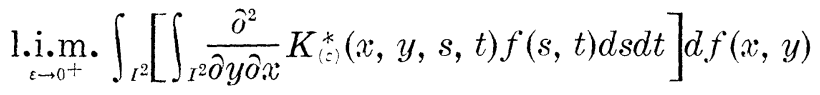

$$
\begin{aligned}
& =\int_{I^{2}}\left[\frac{\partial^{2}}{\partial y \partial x} \int_{I^{2}} K^{*}(x, y, s, t) f(s, t) d s d t\right] d^{*} f(x, y) \quad \text { on } \quad C_{Y} \text {. }
\end{aligned}
$$

Proof. The claim on (6.10) can be treated as that in (6.7). As for (6.11) we may use (5.9) with $H_{i}(x, y, s, t) \equiv \frac{\partial^{2}}{\partial y \partial x} K_{i}(x, y, s, t)$ to see that

$$
\begin{aligned}
\int_{I^{2}} & {\left[\int_{I^{2}} \frac{\partial^{2}}{\partial y \partial x} K_{(\varepsilon)}^{*}(x, y, s, t) f(s, t) d s d t\right] d f(x, y) } \\
= & D(K)\left\{C \int_{I^{2}}\left[\int_{I^{2}} \frac{\partial^{2}}{\partial y \partial x} K_{s}(x, y, s, t) f(s, t) d s d t\right] d f(x, y)\right. \\
& \left.-\int_{I^{2}}\left[\int_{I^{2}}\left(\int_{1^{2}} \frac{\partial^{2}}{\partial y \partial x} K_{:}(x, y, u, v) \gamma(u, v, s, t) d u d v\right) f(s, t) d s d t\right] d f(x, y)\right\},
\end{aligned}
$$

and the first term in the braces converges in the mean to

$$
C \int_{I^{2}}\left[\left(\hat{\partial}^{2} / \partial y \partial x\right) \int_{I^{2}} K(x, y, s, t) f(s, t) d s d t\right] d * f(x, y)
$$

on $C_{Y}$ by the preceding lemma. Therefore, in view of (5.3) and (5.7), it remains to show that

$$
\begin{aligned}
& \underset{\varepsilon \rightarrow 0^{+}}{\lim .} \int_{I^{2}}\left[\int_{I^{2}}\left(\int_{I^{2}} \frac{\partial^{2}}{\partial y \partial x} K_{s}(x, y, u, v) \circlearrowright(u, v, s, t) d u d v\right) f(s, t) d s d t\right] d f(x, y) \\
& =\int_{I^{2}}\left[\frac{\partial^{2}}{\partial y \partial x} \int_{I^{2}}\left(\int_{I^{2}} K(x, y, u, v) \circlearrowright(u, v, s, t) d u d v\right) f(s, t) d s d t\right] d^{*} f(x, y)
\end{aligned}
$$

on $C_{Y}$ whose proof is essentially on the same lines as that of Lemma 8.

LEMMA 9. Let $F(f)$ be a nonnegative Yeh-Wiener measurable functional on $C_{Y}$, and let $K(x, y, s, t)$ satisfy the hypotheses of Theorem $I$. Then under the Fredholm transformation $T$ in (1.1), we have

$$
\int_{C_{Y}} F(g) d_{I^{\prime}} g \geqq|D(K)| \int_{C_{Y}} F(T f) \cdot \exp \{-\Phi(f)\} d_{1} f,
$$

where $\Phi(f)$ is given by (2.8).

Proof. First we assume that $F(f)$ is a bounded nonnegative functional continuous on $C_{Y}$. with respect to the uniform topology, and is dependent only on the $n^{2}$ values of $f$ at $(x, y)=(i / n, j / n) ; i, j=1$, $2, \cdots, n$. Since $\lim _{\varepsilon \rightarrow 0}+D\left(K_{\varepsilon}\right)=D(K) \neq 0$ and $\lim _{n \rightarrow \infty} D\left(K_{\varepsilon(n)}\right)=D\left(K_{\varepsilon}\right)$ 
by Lemma 4 , there exist $\varepsilon^{\prime}$ and $N(\varepsilon)$ such that $0<\varepsilon<\varepsilon^{\prime}$ and $n \geqq N(\varepsilon)$ imply $D\left(K_{\varepsilon}\right) \neq 0$ and $D\left(K_{\varepsilon,(n)}\right) \neq 0$. For such $\varepsilon$ and $n$ we may apply Lemma 2 to obtain

$$
\begin{aligned}
\int_{C_{Y}} F\left(g_{n}\right) d_{Y} g= & \left|\Delta\left(K_{\varepsilon,(n)}\right)\right| \int_{C_{Y}} F\left[f_{(n)}+\int_{I^{2}} K_{\varepsilon,(n)}(\cdot, \cdot, s, t) f_{(n)}(s, t) d s d t\right] \\
& \cdot \exp \left\{-\int_{I^{2}}\left[\int_{I^{2}} \frac{\partial^{2}}{\partial y \partial x} K_{\varepsilon,(n)}(x, y, s, t) f_{(n)}(s, t) d s d t\right]^{2} d x d y\right. \\
& \left.\left.-2 \int_{I^{2}}\left[\int_{I^{2}} \frac{\partial^{2}}{\partial y \partial x} K_{\varepsilon,(n)}(x, y, s, t) f_{(n)}(s, t) d s d t\right] d f_{(n)} x, y\right)\right\} d_{Y} f
\end{aligned}
$$

As $n \rightarrow \infty f_{(n)}(x, y) \rightrightarrows f(x, y)$ and $K_{\varepsilon,(n)}(x, y, s, t) \rightrightarrows K_{\varepsilon}(x, y, s, t)$. Hence $g_{n}(x, y) \equiv f_{(n)}(x, y)+\int_{Y^{2}} K_{\varepsilon,(n)}(x, y, s, t) f_{(n)}(s, t) d s d t \rightrightarrows g(x, y)=f(x, y)+$ $\int_{I^{2}} K_{\varepsilon}(x, y, s, t) f(s, t) d s d t$. Therefore $F\left(g_{n}\right) \rightarrow F(g)$ boundedly. Thus $\lim _{n} \int_{C_{Y}}^{I^{2}} F\left(g_{n}\right) d_{Y} g=\int_{C_{Y}} F(g) d_{Y} g$. Now, by Fatou's lemma it follows from (6.13) with the help of Lemma 3, (4.11), and (4.13) that

$$
\begin{aligned}
\int_{C_{Y}} F(g) d_{Y} g \geqq & \mid D\left(K_{\varepsilon}\right)^{\prime} \int_{C_{Y}} F^{r}\left[f+\int_{I^{2}} K_{\varepsilon}(\cdot, \cdot, s, t) f(s, t) d s d t\right] \\
& \cdot \exp \left\{-\int_{I^{2}}\left[\int_{I^{2}} \frac{\partial^{2}}{\partial y \partial x} K_{\varepsilon}(x, y, s, t) f(s, t) d s d t\right]^{2} d x d y\right. \\
& \left.-2 \int_{I^{2}}\left[\int_{I^{2}} \frac{\partial^{2}}{\partial y \partial x} K_{\varepsilon}(x, y, s, t) f(s, t) d s d t\right] d f(x, y)\right\} d d_{Y} f
\end{aligned}
$$

By $(6.1) \int_{I^{2}} K_{\varepsilon}(x, y, s, t) f(s, t) d s d t \rightrightarrows \int_{I^{2}} K(x, y, s, t) f(s, t) d s d t$ as $\varepsilon \rightarrow 0^{+}$, and hence

$$
F\left[f+\int_{I^{2}} K_{\varepsilon}(\cdot, \cdot, s, t) f(s, t) d s d t\right] \rightarrow F\left[f+\int_{I^{2}} K(\cdot, \cdot, s, t) f(s, t) d s d t\right]
$$

boundedly. Since "mean convergence" implies the existence of an almost everywhere convergent subsequence, it follows from (6.6) that there exists a monotone sequence $\varepsilon_{u} \downarrow 0$ such that

$$
\begin{aligned}
& \lim _{n \rightarrow \infty} \int_{I^{2}}\left[\int_{I^{2}} \frac{\partial^{2}}{\partial y \partial x} K_{\varepsilon_{n}}(x, y, s, t) f(s, t) d s d t\right] d f(x, y) \\
& \quad=\int_{I^{2}}\left[\frac{\partial^{2}}{\partial y \partial x} \int_{I^{2}} K(x, y, s, t) f(s, t) d s d t\right] d^{*} f(x, y) \text { for a.a. } f \in C_{Y}
\end{aligned}
$$

Now we replace $\varepsilon_{n}$ for $\varepsilon$ in (6.14), and then use Fatou's Lemma together with (6.2) and (6.15) to arrive at (6.12). This completes the proof for the case when $F(f)$ in nonnegative, bounded, and continuous in uniform topology. To obtain the result for arbitrary nonnegative measurable 
functionals, we go through, as usual, the following steps: after proving it for the preceding case, we go to characteristic functionals of intervals, then 0 -sets, then $0_{\hat{o}}$-sets, and then mull-sets. (In the last case we get equality rather than inequality, both sides being zero.) Then to characteristic functionals of measurable sets, nonnegative simple functionals, nonnegative bounded Yeh-Wiener measurable functionals, and then finally nonnegative Yeh-Wiener measurable functionals. For the details of these steps see [2: pp. 391-392].

On account of (5.9), (5.10), (6.3), (6.5), and (6.11) we can establish the following on the same lines as above:

Lemma 9'. Let $F(g)$ be a nonnegative Yeh-Wiener measurable functional on $C_{Y}$, and let $K(x, y, s, t)$ be as in Theorem I and $K^{*}(x, y, s, t)$ its resolvent kernel. Then under the transformation

$$
T^{-1}: g(x, y) \rightarrow f(x, y)=g(x, y)+\int_{I^{2}} K^{*}(x, y, s, t) g(s, t) d s d t,
$$

we have

$$
\int_{C_{Y}} F(f) d_{Y} f \geqq\left|D^{-1}(K)\right| \int_{C_{Y}} F\left(T^{-1} g\right) \cdot \exp \left\{-\Phi^{*}(g)\right\} d_{Y} g,
$$

where

$$
\begin{aligned}
\Phi^{*}(g)= & \int_{I^{2}}\left[\frac{\partial^{2}}{\partial x \partial y} \int_{I^{2}} K^{*}(x, y, s, t) g(s, t) d s d t\right]^{2} d x d y \\
& +2 \int_{I^{2}}\left[\frac{\partial^{2}}{\partial y \partial x} \int_{I^{2}} K^{*}(x, y, s, t) g(s, t) d s d t\right] d^{*} g(x, y) .
\end{aligned}
$$

7. Proof of theorems. We prove Theorem I for nonnegative Yeh-Wiener measurable functionals first. In this case by Lemma 9 we have under the transformation $T$ in (1.1),

$$
\int_{C_{Y}} F(g) d_{Y} g \geqq|D(K)| \int_{C_{Y}} F[T f] \exp \{-\Phi(f)\} d_{Y} f,
$$

and upon applying Lemma $9^{\prime}$ on tne right-hand side of (7.1), we obtain under the transformation

$$
\begin{aligned}
& T^{-1}: g(x, y) \rightarrow f(x, y)=g(x, y)+\int_{I^{2}} K^{*}(x, y, s, t) g(s, t) d s d t \\
& \int_{C_{Y}} F[T f] \exp \{-\Phi(f)\} d_{Y} f \\
& \quad \geqq\left|D^{-1}(K)\right| \int_{C_{Y}} F\left(T \circ T^{-1} g\right) \exp \left\{-\Phi\left(T^{-1} g\right)\right\} \cdot \exp \left\{-\Phi^{*}(g)\right\} d_{Y} g .
\end{aligned}
$$


Hence if we show that

$$
\Phi\left(T^{-1} g\right)+\Phi^{*}(g)=0 \text { for a.a. } g \text { in } C_{Y},
$$

then it will follow from (7.1) and (7.2) that

$$
\begin{aligned}
\int_{C_{Y}} F(g) d_{Y} g & \geqq|D(K)| \int_{C_{Y}} F[T f] \exp \{-\Phi(f)\} d_{Y} f \\
& \geqq \int_{C_{Y}} F(g) d_{Y} g
\end{aligned}
$$

and hence the theorem will follow for the case. Observe now that if $T(f)=g$ and so $T^{-1}(g)=f$, then

$$
\begin{aligned}
& \int_{I^{2}} K(x, y, s, t) f(s, t) d s d t=g(x, y)-f(x, y), \\
& \int_{I^{2}} K^{*}(x, y, s, t) g(s, t) d s d t=f(x, y)-g(x, y),
\end{aligned}
$$

and from (3.10) and (3.11), we see that the left-hand side of the first equation in (7.4) is absolutely continuous, and

$$
\frac{\partial^{2}}{\partial y \partial x} \int_{I^{2}} K(x, y, s, t) f(s, t) d s d t
$$

is continuous. Therefore $\left(\partial^{2} / \partial y \partial x\right)[g(x, y)-f(x, y)]$ is also continuous. Therefore by the corollary to Theorem 4 in [3], we see that

$$
\begin{array}{r}
\int \frac{\partial^{2}}{I^{2}}[g(x, y)-f(x, y)] d^{*}[g(x, y)-f(x, y)] \\
\equiv \int_{I^{2}}\left\{\frac{\partial^{2}}{\partial y \partial x}[g(x, y)-f(x, y)]\right\}^{2} d x d y
\end{array}
$$

Hence from (2.8), (7.4), and (7.5) it follows that

$$
\begin{aligned}
\Phi\left(T^{-1} g\right)= & \Phi(f) \\
= & \int_{I^{2}}\left\{\frac{\partial^{2}}{\partial y \partial x}[g(x, y)-f(x, y)]\right\}^{2} d x d y \\
& +2 \int_{I^{2}} \frac{\partial^{2}}{\partial y \partial x}[g(x, y)-f(x, y)] d^{*} f(x, y) \\
= & \int_{I^{2}}\left\{\frac{\partial^{2}}{\partial y \partial x}[g(x, y)-f(x, y)]\right\}^{2} d x d y \\
& +2 \int_{I^{2}} \frac{\partial^{2}}{\partial y \partial x}[g(x, y)-f(x, y)] d^{*} f(x, y) \\
& -2 \int_{I^{2}}\left\{\frac{\partial^{2}}{\partial y \partial x}[g(x, y)-f(x, y)]\right\}^{2} d x d y,
\end{aligned}
$$


and from (6.16) and (7.4)

$$
\begin{aligned}
\Phi^{*}(g)= & \int_{I^{2}}\left\{\frac{\partial^{2}}{\partial y \partial x}[f(x, y)-g(x, y)]\right\}^{2} d x d y \\
& +2 \int_{I^{2}} \frac{\partial^{2}}{\partial y \partial x}[f(x, y)-g(x, y)] d^{*} g(x, y) .
\end{aligned}
$$

Therefore (7.3) follows from (7.6) and (7.7). Hence the theorem holds for any nonnegative Yeh-Wiener measurable functionals. For arbitrary real Yeh-Wiener measurable functionals the theorem will also hold by considering the positive part and the negative part separately. For complex functionals we get the same result once we consider real part and imaginary part separately. This completes the proof of Theorem I.

To prove Theorem II we consider the following two transformations

$$
\begin{aligned}
& I_{1}: f(x, y) \rightarrow g(x, y)=f(x, y)+f_{0}(x, y), \\
& I_{2}: f(x, y) \rightarrow h(x, y)=f(x, y)+\int_{I^{2}} K(x, y, s, t) f(s, t) d s d t .
\end{aligned}
$$

The theorem now follows by the use of Cameron-Martin translation theorem (see [11] or more precisely Theorem 1.4 in [4] on $L_{1}$ and then our Theorem I for $L_{2}$.

REMARK. As mentioned in the introduction our theorems in onedimensional version have slightly different forms from the ones given in [1], the difference being in the expressions of $\Phi(f)$ and $\Psi(f)$. The $\Phi(f)$ in [1] is given by

$$
\begin{aligned}
\Phi(f)= & \int_{0}^{1}\left[\frac{d}{d x} \int_{0}^{1} K(x, s) f(s) d s\right]^{2} d x \\
& +2 \int_{0}^{1}\left[\int_{0}^{1} \frac{\partial}{\partial x} K(x, s) f(s) d s\right] d f(x)+\int_{0}^{1} J(x) d\left[f^{2}(x)\right],
\end{aligned}
$$

and ours is in the form

$$
\Phi(f)=\int_{0}^{1}\left[\frac{d}{d x} \int_{0}^{1} K(x, s) f(s) d s\right]^{2} d x+2 \int_{0}^{1}\left[\frac{d}{d x} \int_{0}^{1} K(x, s) f(s) d s\right] d^{*} f(x) .
$$

However by the assumptions given on $K(x, s)$ in [1], we have that

$$
\begin{aligned}
\frac{d}{d x} \int_{0}^{1} K(x, s) f(s) d s= & \left.\frac{d}{d x} \int_{0}^{x} K_{2}(x, s) f(s) d s+\int_{x}^{1} K_{1}(x, s) f(s) d s\right] \\
& =\left[K_{2}(x, x)-K_{1}(x, x)\right] f(x)+\int_{0}^{1} \frac{\partial}{\partial x} K(x, s) f(s) d s
\end{aligned}
$$




$$
=J(x) f(x)+\int_{0}^{1} \frac{\partial}{\partial x} K(x, s) f(s) d s .
$$

But assuming absolute continuity of $J(x)$ with $J^{\prime}(x) \in L^{2}$, it follows from Theorem 5 in [3] that

$$
\int_{0}^{1} J(x) f(x) f(x) d^{*} f(x)=\frac{1}{2} \int_{0}^{1} J(x) d\left[f^{2}(x)\right] \text { for a.a. } f \in C_{W} \text {. }
$$

Also as mentioned earlier

$$
\int_{0}^{1}\left[\int_{0}^{1} \frac{\partial}{\partial x} K(x, s) f(s) d s\right] d^{*} f(x)=\int_{0}^{1}\left[\int_{0}^{1} \frac{\partial}{\partial x} K(x, s) f(s) d s\right] d f(x)
$$

for almost all $f$ in $C_{W}$. Thus (7.8) and (7.9) represent essentially the same thing.

\section{REFERENCES}

1. R. H. Cameron and W. T. Martin, Transformations of Wiener integrals under a general class of linear transformations, Trans. Amer. Math. Soc., 58 (1945), 184-219. 2. - On transformations of Wiener integrals under transformations, Ann. of Math., 45 (1944), 386-396.

3. C. Park, A generalized Paley-Wiener-Zygmund integral and its applications, Proc. Amer. Math. Soc., 23 (1969), 388-400.

4. - Generalized Riemann-Stieltjes integral over the K.Y.W. space of functions of two variables, Thesis, Univ. of Minnesota (1968).

5. I. E. Segal, Distributions in Hilbert space and canonical systems of operators, Trans. Amer. Math. Soc., 88 (1958), 12-41.

6. - Algebraic integration theory, Bull. Amer. Math. Soc., 71 (1965), 419-489.

7. L. A. Shepp, Radon-Nikodym derivatives of Gaussian measures, Ann. Math. Statist.,

37 (1966), 321-354.

8. F. G. Tricomi, Integral equations, Interscience Publishers (1957).

9. D. A. Woodward, A general class of linear transformations of Wiener integrals, Trans. Amer. Math. Soc., 100 (1961), 459-480.

10. J. Yeh, Wiener measure in a space of functions of two variables, Trans. Amer. Math. Soc., 95 (1960), 433-450.

11. - Cameron-Martin translation theorems in the Wiener space of functions of two variables, Trans. Amer. Math. Soc., 107 (1963), 1427-1436.

12. - Orthogonal development of functionals and related theorems in the Wiener space of functions of two variables, Pacific J. Math., 13 (1963), 1427-1436.

Received October 23, 1970. This research was performed at Miami University and the U.S.A.F. Aerospace Research Laboratories while in the capacity of an Ohio State University Research Foundation visiting Research Associate under Contract F33615 67 C 1758.

Miami University 



\section{PACIFIC JOURNAL OF MATHEMATICS}

\section{EDITORS}

H. SAMELSON

Stanford University

Stanford, California 94305

C. R. HOBBY

University of Washington

Seattle, Washington 98105
J. DugundJI

Department of Mathematics

University of Southern California

Los Angeles, California 90007

RICHARD ARENS

University of California

Los Angeles, California 90024

\section{ASSOCIATE EDITORS}

E. F. BeCKENBACH

B. H. NeumanN

F. WOLF

K. YosHIDA

\section{SUPPORTING INSTITUTIONS}

UNIVERSITY OF BRITISH COLUMBIA

CALIFORNIA INSTITUTE OF TECHNOLOGY

UNIVERSITY OF CALIFORNIA

MONTANA STATE UNIVERSITY

UNIVERSITY OF NEVADA

NEW MEXICO STATE UNIVERSITY

OREGON STATE UNIVERSITY

UNIVERSITY OF OREGON

OSARA UNIVERSITY
UNIVERSITY OF SOUTHERN CALIFORNIA STANFORD UNIVERSITY

UNIVERSITY OF TOKYO

UNIVERSITY OF UTAH

WASHINGTON STATE UNIVERSITY

UNIVERSITY OF WASHINGTON

AMERICAN MATHEMATICAL SOCIETY

NAVAL WEAPONS CENTER

Printed in Japan by International Academic Printing Co., Ltd., Tokyo, Japan 


\section{Pacific Journal of Mathematics}

\section{Vol. 40, No. $1 \quad$ September, 1972}

Alex Bacopoulos and Athanassios G. Kartsatos, On polynomials

approximating the solutions of nonlinear differential equations........

Monte Boisen and Max Dean Larsen, Prüfer and valuation rings with zero

divisors ..........................................

James J. Bowe, Neat homomorphisms

David W. Boyd and Hershy Kisilevsky, The Diophantine equation

$$
u(u+1)(u+2)(u+3)=v(v+1)(v+2) \ldots \ldots \ldots \ldots \ldots \ldots \ldots
$$

George Ulrich Brauer, Summability and Fourier analysis ...............

Robin B. S. Brooks, On removing coincidences of two maps when only one,

rather than both, of them may be deformed by a homotopy ............

Frank Castagna and Geert Caleb Ernst Prins, Every generalized Petersen

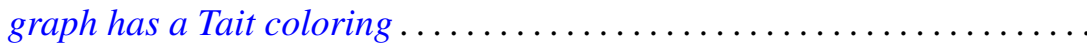

Micheal Neal Dyer, Rational homology and Whitehead products ..........

John Fuelberth and Mark Lawrence Teply, The singular submodule of a

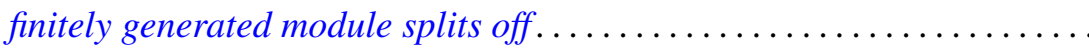

Robert Gold, $\Gamma$-extensions of imaginary quadratic fields ............ 83

Myron Goldberg and John W. Moon, Cycles in k-strong tournaments.......

Darald Joe Hartfiel and J. W. Spellmann, Diagonal similarity of irreducible

matrices to row stochastic matrices...............

Wayland M. Hubbart, Some results on blocks over local fields ..

Alan Loeb Kostinsky, Projective lattices and bounded homomorphisms....

Kenneth O. Leland, Maximum modulus theorems for algebras of operator

valued functions ...

Jerome Irving Malitz and William Nelson Reinhardt, Maximal models in the

language with quantifier "there exist uncountably many" ..

John Douglas Moore, Isometric immersions of space forms in space

forms.

Ronald C. Mullin and Ralph Gordon Stanton, A map-theoretic approach to

Davenport-Schinzel sequences ....................

Chull Park, On Fredholm transformations in Yeh-Wiener space. .

Stanley Poreda, Complex Chebyshev alterations ..............

Ray C. Shiflett, Extreme Markov operators and the orbits of Ryff. ...

Robert L. Snider, Lattices of radicals .....................

Ralph Richard Summerhill, Unknotting cones in the topological

category ................................

Charles Irvin Vinsonhaler, A note on two generalizations of $\mathrm{QF}-3 \ldots \ldots 229$

William Patterson Wardlaw, Defining relations for certain integrally

parameterized Chevalley groups...................

William Jennings Wickless, Abelian groups which admit only nilpotent

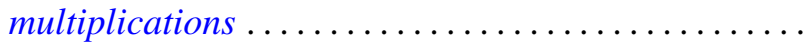

